\title{
Chiral-symmetry breaking in dual QCD
}

\author{
M. Baker \\ Department of Physics, University of Washington, Seattle, Washington 98195 \\ J. S. Ball \\ Department of Physics, University of Utah, Salt Lake City, Utah 84112 \\ F. Zachariasen \\ California Institute of Technology, Pasadena, California 91125
}

(Received 25 April 1988)

\begin{abstract}
In the context of the formulation of QCD with dual potentials, we show that chiral-symmetry breaking occurs only in the confined state. Therefore, the transition temperature, beyond which chiral symmetry is restored, is the same as the deconfinement temperature. To carry out the calculation, it is necessary to couple quarks to dual gluons. We indicate how this is done (to lowest order in the magnetic coupling constant) and give the Feynman rules for quark-dual-gluon vertices.
\end{abstract}

\section{INTRODUCTION}

This paper is devoted to a study of chiral-symmetry breaking and the chiral phase transition in the context of dual QCD (Ref. 1). Dual QCD is ordinary QCD expressed in terms of dual potentials $C_{\mu}^{a}$ rather than the usual $A_{\mu}^{a}$. The dual potentials are the variables most appropriate for discussing the long-range confining regime of QCD.

It has often been suggested that confinement and chiral-symmetry breaking are intimately connected, ${ }^{2}$ and indeed lattice QCD calculations ${ }^{3}$ strongly support this view, as do the calculations we shall describe here. We shall study chiral-symmetry breaking within a simplified approximation, but which we feel nevertheless includes most of the important physics. It uses the Dyson equation for the quark propagator, and the "rainbow graph" approximation, and furthermore looks only for a constant, momentum-independent, chiral-symmetrybreaking quark mass. Within this approximation, we find that there is no chiral-symmetry breaking unless there is also confinement.

Confinement in QCD occurs, as in dual superconductivity, because of spontaneous symmetry breaking, in which the superconducting or confining vacuum consists of a magnetic condensate, having a nonzero value of the color-magnetic field squared. In the perturbative, or nonconfining vacuum, there is no chiral breakdown. Only when the condensate is present does chiralsymmetry breaking take place. This means that there can be no chiral breaking above the deconfinement temperature $T_{c}$. Chiral breaking in the nonperturbative vacuum is described by a gap equation much like the one encountered in superconductivity. ${ }^{4}$ The role of the Debye energy is played by the magnetic condensate. In QCD the condensate vanishes at the deconfinement temperature $T_{c}$, at which point chiral symmetry is restored. In superconductivity, in contrast, the Debye energy never van- ishes; instead the gap disappears at the critical temperature determined by the solution of the gap equation. ${ }^{4}$

In I we have constructed the long-range limit of the dual Lagrangian $\mathcal{L}(C)$ describing QCD as a function of the dual potentials. We expect $\mathcal{L}(C)$ to be valid at low energies, which means energies (or temperatures) up to a scale $M$ which we have estimated in I to be of order 450 $\mathrm{MeV}$. Beyond this scale, higher-order corrections to our approximate $\mathcal{L}(C)$ become significant.

$\mathcal{L}(C)$ was explicitly constructed to describe the confining regime of QCD, that is, temperatures below the deconfinement point. Deconfinement is estimated to occur in the range 100-200 MeV. This is well below $M$, and therefore it is possible that $\mathcal{L}(C)$ will continue to be valid in a region above the deconfinement point. If this is true we arrive at the following picture: In the confined regime, there are only short-range correlations among dual gluons, so the dual Wilson loop obeys a perimeter law and the usual Wilson loop an area law; hence confinement. Above $T_{c}$, there are long-range correlations among dual gluons [i.e., the dual gluon propagator contains massless singularities, see Eq. (2.27)] so that the area law for the Wilson loop no longer holds. Nevertheless, there are still effects due to the mutual interactions of dual gluons, expressed through the fact that the dielectric constant $\epsilon$ is not unity. The usual asymptotically free perturbation theory is applicable only above the scale $M$, where $\epsilon$ becomes one. In this paper we shall assume the validity of $\mathcal{L}(C)$ up to the scale $M$, so that $\mathcal{L}(C)$ can be used to describe QCD both in the confined regime and in the deconfined regime not too far above the deconfinement temperature.

Section III of this paper discusses the perturbative vacuum case and, within the simplifying assumptions used, shows that there is no chiral-symmetry-breaking solution. Section IV describes the nonperturbative confining case, and derives the analog of the gap equation. The Appendix elaborates on the results given in both of these sections. 
Before any analysis of chiral-symmetry breaking can be carried out, however, it is of course necessary to introduce quarks into dual QCD. Section II is devoted to this issue. Because of the use of dual potentials, quarks are awkward to deal with, as are monopoles in ordinary QED. In Sec. II we derive, in lowest order in the magnetic coupling constant $g$, the Feynman rules for coupling quarks to dual gluons and to the other fields appearing in the dual QCD Lagrangian. Using these rules, we also derive some useful formulas necessary for writing down the Dyson equation for the quark propagator.

\section{COUPLING QUARKS TO DUAL GLUONS}

Our first task is to introduce quarks into the dual Lagrangian $\mathcal{L}(C)$. At the Abelian level, this is the same problem as coupling magnetic monopoles to ordinary photons in quantum electrodynamics, and we begin with that.

First, suppose we have a stationary point monopole with magnetic charge $g$ located at the origin. The relevant Maxwell equation is

$$
\boldsymbol{\nabla} \cdot \mathbf{B}=g \delta^{3}(\mathbf{x})
$$

This equation is incompatible with the usual relation between the magnetic field and the vector potential, which is

$$
\mathbf{B}=\boldsymbol{\nabla} \times \mathbf{A} .
$$

Equation (2.2) insists that there is no net magnetic flux through any closed surface; the way this is implemented for a Dirac monopole, for example, is by attaching a string to the monopole, which brings in just as much flux as leaves the monopole in its Coulomb field. Since (2.1) requires that there be net flux issuing from the monopole, (2.2) cannot be retained.

The resolution ${ }^{5}$ is to change (2.2) by adding a field canceling the string in $\boldsymbol{\nabla} \times \mathbf{A}$. Thus we replace $(2.2)$ by

$$
\mathbf{B}=\boldsymbol{\nabla} \times \mathbf{A}+\mathbf{B}_{s} .
$$

The "string field" $\mathbf{B}_{s}$ is chosen to satisfy

$$
\boldsymbol{\nabla} \cdot \mathbf{B}_{s}=g \delta^{3}(\mathbf{x}),
$$

to which the string-field solution is

$$
\mathbf{B}_{s}=-g \delta(x) \delta(y) \theta(-z) \widehat{\mathbf{e}}_{z},
$$

if the string is chosen to lie along the negative $z$ axis.

The dynamics is contained in the Maxwell equation

$$
\boldsymbol{\nabla} \times \mathbf{B}=0 \text {. }
$$

This equation determines the vector potential $\mathbf{A}$. In the $\boldsymbol{\nabla} \cdot \mathbf{A}=0$ gauge, we have

$$
-\nabla^{2} \mathbf{A}+\boldsymbol{\nabla} \times \mathbf{B}_{s}=0 ;
$$

the solution to (2.7) is the Dirac string field

$$
\mathbf{A}=\frac{g}{4 \pi} \frac{1-\cos \theta}{r \sin \theta} \widehat{\mathbf{e}}_{\phi},
$$

in spherical coordinates. Finally, Eqs. (2.3), (2.5), and
(2.8) give for the magnetic field $\mathbf{B}$ precisely the Coulomb field, with no string:

$$
\mathbf{B}=\frac{g}{4 \pi r^{2}} \widehat{\mathbf{e}}_{r} .
$$

The generalization of this simple procedure to incorporate relativity is straightforward. Here, expressing the field tensor $F_{\mu \nu}$ in terms of the vector potential $A_{\mu}$, through

$$
F_{\mu v}=\partial_{\mu} A_{v}-\partial_{v} A_{\mu},
$$

guarantees that the pair of Maxwell equations

$$
-\frac{1}{2} \partial_{v} \epsilon_{\mu \nu \lambda \sigma} F_{\lambda \sigma}=0
$$

is automatically satisfied. However, when there are magnetic sources, the right-hand side of this equation is no longer zero, but is a magnetic current $j_{\mu}^{M}$, so the choice (2.10) is no longer compatible with it. Again the resolution 5 is to modify (2.10) by adding a "string field" $F_{s \mu \nu}$, so that

$$
F_{\mu v}=\partial_{\mu} A_{v}-\partial_{v} A_{\mu}+F_{s \mu v},
$$

and choosing the string field to satisfy

$$
-\frac{1}{2} \partial_{\nu} \epsilon_{\mu \nu \lambda \sigma} F_{s \lambda \sigma}=j_{\mu}^{M} \text {. }
$$

Physically, the $\partial_{\mu} A_{v}-\partial_{v} A_{\mu}$ part of the field tensor in (2.12) contains strings. The string field $F_{s \mu v}$ is simply chosen to cancel these, as in the Coulomb case described above.

The solution to (2.13) is

$$
F_{s \mu \nu}=\frac{1}{n \cdot \partial} \epsilon_{\mu \nu \lambda \sigma} n_{\lambda} j_{\sigma}^{M}
$$

where $n_{\mu}$ gives the string direction.

For a spin $-\frac{1}{2}$ monopole described by a Dirac field $\psi$, the magnetic current is

$$
j_{\sigma}^{M}=g \bar{\psi} \gamma_{\sigma} \psi
$$

where $g$ is the monopole strength. (In a theory with both monopoles and electric charges, compatibility with gauge invariance requires that $e g=2 \pi$.)

The electrodynamic Lagrangian is

$$
\mathcal{L}=-\frac{1}{4} F_{\mu \nu} F_{\mu \nu}
$$

with the decomposition (2.12) there are, therefore, two types of interaction terms. The first comes from the cross term between $\partial_{\mu} A_{v}-\partial_{v} A_{\mu}$ and $F_{s \mu v}$; it gives rise to the graph illustrated in Fig. 1(a), and has a vertex

$$
\Gamma_{v}=g q_{\mu} \frac{\epsilon_{\mu v \lambda \sigma} n_{\lambda} \gamma_{\sigma}}{q \cdot n} .
$$

From the $-\frac{1}{4} F_{s \mu \nu} F_{s \mu v}$ term in $\mathcal{L}$, there is also a fourfermion vertex, shown in Fig. 1(b), for which the Feynman rule is to write

$$
\Gamma=-\frac{1}{2} g^{2} \epsilon_{\mu \nu \alpha \beta} n_{\alpha} \gamma_{\beta}^{(1)} \epsilon_{\mu \nu \gamma \delta} n_{\gamma} \gamma_{\delta}^{(2)} .
$$

Since the quadratic (in $A$ ) part of $\mathcal{L}$ is unchanged, the usual Feynman rule for the photon propagator still ob- 


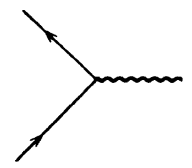

(a)

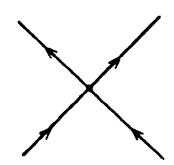

(b)
FIG. 1. Feynman diagrams for the interaction of monopoles (solid lines) and ordinary photons (wavy lines).

tains.

It is a straightforward matter to add up the graphs shown in Fig. 2, for example, and verify that the usual scattering amplitude is obtained for monopole-monopole scattering.

To couple quarks to dual gluons in dual QCD we may try to follow an analogous procedure. The Maxwell equation (2.13) should now be replaced by

$$
-\frac{1}{2} D_{\nu}(C) \epsilon_{\mu \nu \lambda \sigma} G_{s \lambda \sigma}=k_{\mu} .
$$

To lowest order, $D_{v}(C) \rightarrow \partial_{v}$ and $k_{\mu} \rightarrow j_{\mu}=e \bar{q} \gamma_{\mu} q$; thus to lowest (zeroth) order in $g$, the magnetic charge, there is a dual-gluon-quark vertex such as in Fig. 1(a) and a fourquark vertex such as in Fig. 1(b), coming from the $-\frac{1}{4} G_{\mu \nu} G_{\mu \nu}$ term in the dual Lagrangian. ${ }^{1}$ There is also a coupling of the quark to the $\widetilde{F}$ field, coming from the $\frac{1}{2} M \widetilde{F}_{\mu \nu} G_{\mu \nu}$ term in $\mathcal{L}(C)$. All three vertices, with their Feynman rules, are shown in Fig. 3.

In higher orders in $g$, however, complications arise. Because of the nonlinear covariant derivative $D_{v}(C)$, an infinite series of vertices of order $e g^{n}, n=0,1,2, \ldots$, is generated. Furthermore, we do not know what the "current" $k_{\mu}$ in Eq. (2.19) is, beyond lowest order. It must be a quantity transforming according to the magnetic, not the electric, gauge group, so it cannot coincide with $j_{\mu}$ except in lowest order.

Dual QCD necessarily involves both $e$ and $g$; it is a theory in which both electric and magnetic charges must exist. As in electrodynamics, this requires the Dirac quantization condition $e g=2 \pi$ to hold, and, consequently, destroys any possibility of doing perturbation theory: if expressed in terms of either $e$ (or $g$ ), the perturbation series for any process involves all powers of $e$, positive and negative. Further, any given power of $e$ is not associated with graphs having a given number of loops. As a result, adding up all graphs with a given number of loops does not, in general, give a gauge-invariant result.

All of these difficulties mean that we do not know how to do higher-order calculations with quarks in dual QCD.

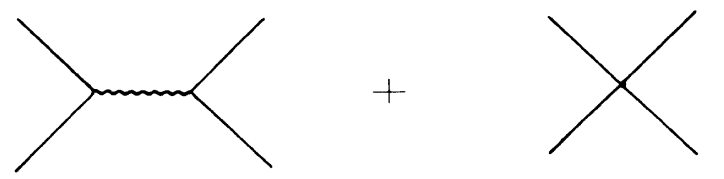

FIG. 2. Monopole-monopole scattering graphs. The two graphs add up to give the usual $\gamma_{\mu}^{(1)}\left(1 / q^{2}\right) \gamma_{\mu}^{(2)}$ interaction.

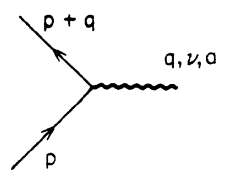

$$
\mathrm{eq}_{\mu} \frac{\epsilon_{\mu \nu \lambda \sigma} n_{\lambda} \gamma_{\sigma}}{\mathrm{q} \cdot \mathrm{n}} \quad \frac{\lambda^{\circ}}{2}
$$
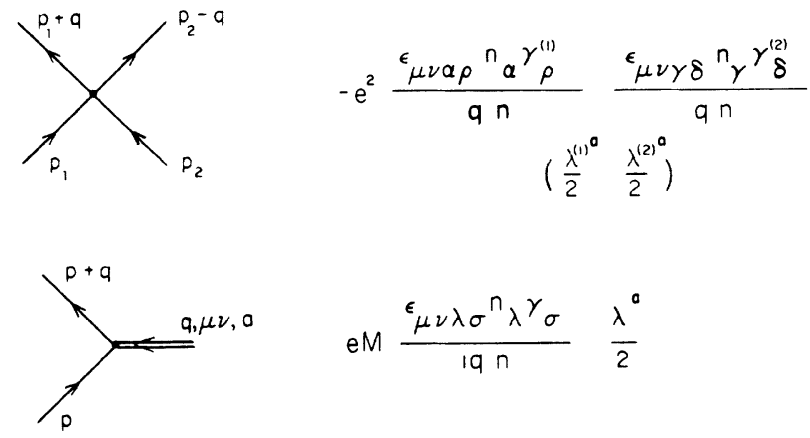

$\mathrm{eM} \frac{\epsilon_{\mu \nu \lambda \sigma^{n} \lambda^{\gamma} \sigma}}{19 n} \quad \frac{\lambda^{0}}{2}$

FIG. 3. Feynman rules, to lowest order in the magnetic coupling constant $g$, for coupling quarks to dual gluons and to the $\widetilde{F}$ field.

We will confine ourselves to the lowest-order graphs of Fig. 3.

Using these rules, we can now demonstrate that in lowest order in $g$, dual QCD reproduces the result of using ordinary $A$ language QCD. Lowest order in $g$ means the Abelian approximation. As explained in $I$, at the Abelian level the Lagrangian

$$
\mathcal{L}(A)=-\frac{1}{4} F_{\mu v}\left(\frac{\square}{\square+M^{2}}\right) F_{\mu v},
$$

where

$$
F_{\mu v}=\partial_{\mu} A_{v}-\partial_{v} A_{\mu},
$$

is exactly equivalent to

$$
\mathcal{L}(C)=-\frac{1}{4} G_{\mu v}\left(\frac{M^{2}}{\square}+1\right) G_{\mu v},
$$

where

$$
G_{\mu v}=\partial_{\mu} C_{v}-\partial_{v} C_{\mu} .
$$

When (2.22) is turned into a local Lagrangian, the Abelian approximation to the dual QCD Lagrangian ${ }^{1}$ [not including the counterterms $W(\widetilde{F})$ required by renormalizability] results:

$$
\mathcal{L}(C)=\frac{M}{2} \widetilde{F}_{\mu \nu} G_{\mu \nu}+\frac{1}{4} \widetilde{F}_{\mu \nu} \square \widetilde{F}_{\mu \nu}-\frac{1}{4} G_{\mu \nu} G_{\mu \nu} .
$$

In (2.20) quarks are introduced in the usual way, by adding a term $-j_{\mu}^{a} A_{\mu}^{a}$, where $j_{\mu}^{a}=e \bar{q}\left(\lambda^{a} / 2\right) \gamma_{\mu} q$. In (2.24), quarks are added by replacing (2.23) with

$$
G_{\mu v}=\partial_{\mu} C_{v}-\partial_{\nu} C_{\mu}+G_{s \mu v}
$$

To order $e^{2}$, both (2.20) and (2.24) should therefore give the same result. That this is in fact so can be verified 
by direct calculation. Adding all of the graphs (for offshell quarks) shown in Fig. 4, with the rules for quark vertices given above, and taking the $C$ and $\widetilde{F}$ propagators from I, Eqs. (3.15)-(3.17), results in the amplitude

$$
\begin{aligned}
& e^{2}\left(1 / q^{2}-M^{2} / q^{4}\right) \\
& \quad \times \gamma_{\mu}^{(1)}\left(\delta_{\mu \nu}-\frac{q_{\mu} n_{v}+q_{\nu} n_{\mu}}{q \cdot n}+\frac{q_{\mu} q_{v} n^{2}}{(q \cdot n)^{2}}\right] \gamma_{v}^{(2)},
\end{aligned}
$$

where $n_{v}$ is the string direction in $G_{s \mu v}$. This is precisely what would have been obtained from (2.20) in axial gauge, where $n_{v}$ is the direction of the axial gauge choice.

As explained in I, however, the Lagrangian (2.24) requires the addition of counterterms for renormalizability; one adds $-W(\widetilde{F})$ to $(2.24)$, where $W(\widetilde{F})$ is given in Eq. (3.22) of $\mathrm{I}$. The dual Lagrangian now contains an additional quadratic term in $\widetilde{F}^{2}$, which modifies the propagators. Thus, including $W(\widetilde{F})$ in $\mathcal{L}(C)$, and relative to the perturbative vacuum, the propagators (3.15)-(3.17) of I are replaced by (in the Landau gauge)

$\Delta_{\mu \nu}^{a b}(q)=\delta^{a b}\left[\delta_{\mu \nu}-\frac{q_{\mu} q_{\nu}}{q^{2}}\right)\left(\frac{q^{2}-M^{* 2}}{q^{2}\left(q^{2}-M^{2}-M^{* 2}\right)}\right)$,

$\Delta_{\mu, \alpha \beta}^{a b}(q)=\delta^{a b}\left(\delta_{\mu \alpha} q_{\beta}-\delta_{\mu \beta} q_{\alpha}\right)\left(\frac{-i M}{q^{2}\left(q^{2}-M^{2}-M^{* 2}\right)}\right)$,

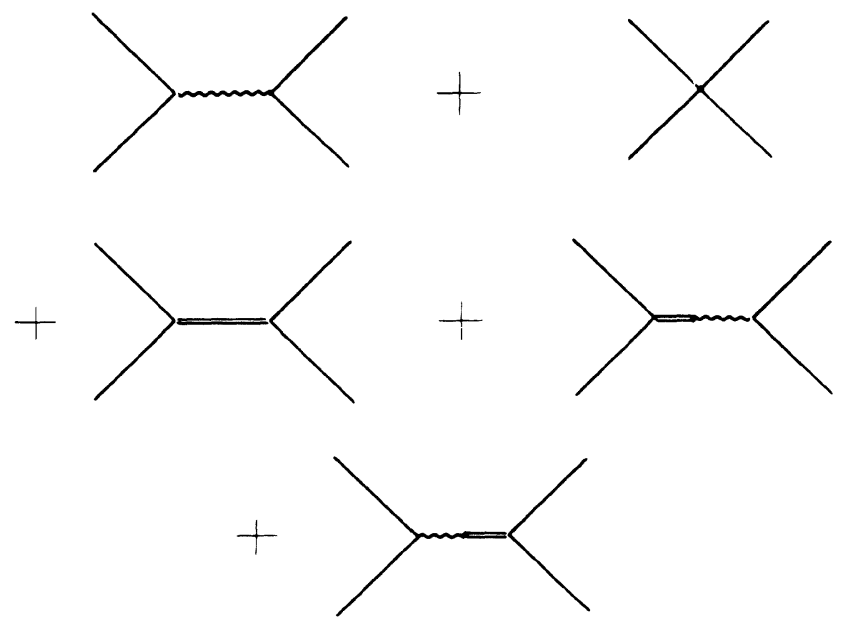

FIG. 4. Feynman graphs for quark-quark scattering to lowest order in $g$. Dual gluons (wavy lines), the $\widetilde{F}$ field (double lines), and the mixed $C-\widetilde{F}$ propagator can be exchanged, and there is also a contribution from the four-quark vertex.

$$
\begin{aligned}
\Delta_{\alpha \beta, \gamma \delta}^{a b}(q)=\delta^{a b}\left[\frac{1}{q^{2}-M^{* 2}}\left(\delta_{\alpha \gamma} \delta_{\beta \gamma}-\delta_{\alpha \delta} \delta_{\beta \gamma}\right)\right. \\
+\frac{M^{2}}{\left(q^{2}-M^{* 2}\right)\left(q^{2}-M^{2}-M^{* 2}\right)} \\
\quad \times\left[\frac{q_{\alpha} q_{\gamma}}{q^{2}} \delta_{\beta \delta}-\frac{q_{\alpha} q_{\delta}}{q^{2}} \delta_{\beta \gamma}\right. \\
\left.\left.\quad-\frac{q_{\beta} q_{\gamma}}{q^{2}} \delta_{\alpha \delta}+\frac{q_{\beta} q_{\delta}}{q^{2}} \delta_{\alpha \gamma}\right]\right],
\end{aligned}
$$

where

$$
M^{* 2} \equiv N \mu^{2}
$$

At $T=0, \mu^{2}<0$ so that the mass $M^{* 2}$ would represent a ghost. But the perturbative vacuum propagators are applicable only above the deconfinement temperature. This is by definition precisely the temperature at which the temperature-dependent effective $\mu^{2}(T)$ changes sign. Thus $M^{* 2}$ is actually positive (and temperature dependent, vanishing at $T=T_{c}$ ).

The graphs of Fig. 4, giving the effective interaction between quarks, may now be recalculated using the perturbative vacuum propagators with $W$ included, Eqs. (2.27)-(2.29). We find, in place of (2.26), the result

$e^{2}\left(\frac{q^{2}-M^{2}-M^{* 2}}{q^{2}\left(q^{2}-M^{* 2}\right)}\right)$

$$
\times \gamma_{\mu}^{(1)}\left(\delta_{\mu v}-\frac{q_{\mu} n_{v}+q_{v} n_{\mu}}{q \cdot n}+\frac{q_{\mu} q_{v} n^{2}}{(q \cdot n)^{2}}\right] \gamma_{v}^{(2)} \text {. }
$$

The $M^{2} / q^{4}$ term is no longer present, reflecting the fact that the ordinary Wilson loop $W_{A}$ no longer obeys an area law. Note that both the effective $A$ gluon propagator (2.31) and the $C$ gluon propagator (2.27) have firstorder poles at $q^{2}=0$.

Equations (2.27)-(2.29) and (2.31) are for the perturbative vacuum. In the nonperturbative vacuum, the magnetic condensate $\left\langle\widetilde{F}_{\mu \nu}^{a} \widetilde{F}_{\mu \nu}^{a}\right\rangle$ takes on a nonzero value $\widetilde{F}_{0}^{2}$, reflecting spontaneous symmetry breaking, and the existence of a dual superconducting state with color-electric confinement. When $\widetilde{F}_{0}^{2} \neq 0$, there are further quadratic terms in $\mathcal{L}(C)$, coming both from the $\widetilde{F}_{\mu v} \mathcal{D}^{2}(C) \widetilde{F}_{\mu v}$ term and from the expansion of $W(\widetilde{F})$ around the nonperturbative value of $\widetilde{F}^{2}$. These terms alter the propagators $\Delta_{\mu \nu}^{a b}, \Delta_{\mu, \alpha \beta}^{a b}$, and $\Delta_{\alpha \beta, \gamma \delta}^{a b}$.

The effect is to remove all massless singularities. When the nonperturbative vacuum propagators are used to compute the effective quark-quark interaction, via Fig. 4, the simple structure of (2.26) or of (2.31) is lost. There is an additional term in the effective propagator of the form

$$
\frac{1}{(n \cdot q)^{2}}\left(\delta_{\mu v}-n_{\mu} n_{v} / n^{2}\right) \text {. }
$$

It is this term which is essential for chiral-symmetry breaking, as we shall see below. 


\section{CHIRAL-SYMMETRY BREAKING: THE PERTURBATIVE VACUUM}

The chiral order parameter $\langle\bar{q} q\rangle$ vanishes unless the quark propagator $S(p)$ has a mass term:

$$
\langle\bar{q} q\rangle=\int \frac{d^{4} p}{(2 \pi)^{4}} \operatorname{Tr} S(p) .
$$

If we write

$$
S(p)=\frac{Z(p)}{\not p-m(p)},
$$

then

$$
\langle\bar{q} q\rangle=\int \frac{d^{4} p}{(2 \pi)^{4}} \frac{Z(p) m(p)}{p^{2}-m^{2}(p)} .
$$

We can study the presence or absence of a mass term through the Dyson equation for $S$; this equation, in dual QCD, is displayed graphically in Fig. 5. The right-hand side of the Dyson equation involves the exact vertices coupling quarks to dual gluons. As previously explained, these couplings are known only to lowest order in $g$. We therefore replace the exact vertices by bare vertices; this is the so-called "rainbow" approximation. To the extent that long-distance phenomena dominate, the exact dual

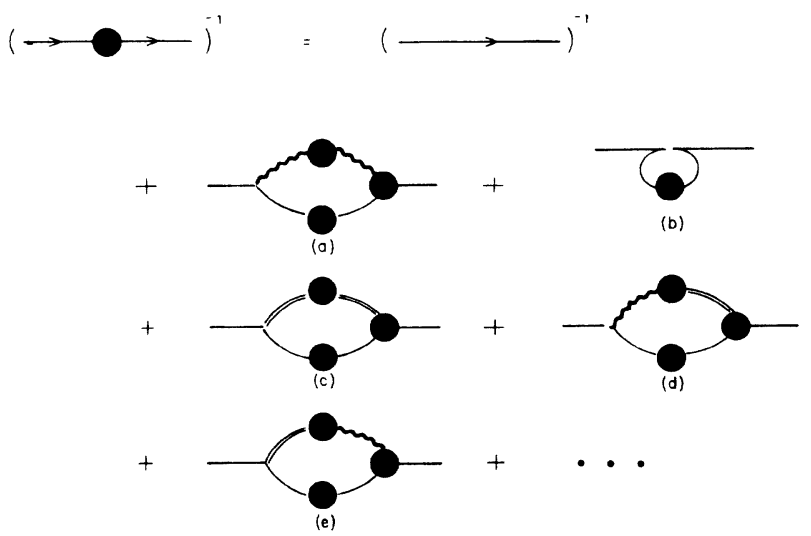

FIG. 5. Graphs contributing to the Dyson equation for the quark propagator. Wavy lines indicate dual gluons; the double line is the $\widetilde{F}$ propagator. The diagram labeled (b) is the contribution of the four-quark interaction; it is the graph that gives rise to the analog of the gap equation in superconductivity. The $+\cdots$ refers to higher-order (in $g$ ) quark vertices.

gluon and $\widetilde{F}$ propagators in the Dyson equation can also be replaced by their lowest-order values. Then, using the results of Sec. II, in the perturbative vacuum the explicit Dyson equation is

$$
S^{-1}(p)=\not p-i e^{2} C_{F} \int d k\left(\frac{k^{2}-M^{2}-M^{* 2}}{k^{2}\left(k^{2}-M^{* 2}\right)}\right) \gamma_{\mu} S(p-k) \gamma_{\nu}\left(\delta_{\mu v}-\frac{k_{\mu} n_{v}+k_{v} n_{\mu}}{(k \cdot n)}+\frac{k_{\mu} k_{n} n^{2}}{(k \cdot n)^{2}}\right) .
$$

Here $n_{v}$ is the string direction, as explained in Sec. II (Ref. 6). Also, $C_{F}=\left(\lambda^{a} / 2\right) \cdot\left(\lambda^{a} / 2\right)=\left(N^{2}-1\right) / 2 N$ for $\mathrm{SU}(N)$.

To look for a nonzero-quark-mass term, we take the trace of (2.4):

$$
\frac{m(p)}{Z(p)}=-i e^{2} C_{F} \int d k\left(\frac{k^{2}-M^{2}-M^{* 2}}{k^{2}\left(k^{2}-M^{* 2}\right)}\right) \frac{m(p-k) Z(p-k)}{(p-k)^{2}-m^{2}(p-k)}\left(2+\frac{k^{2} n^{2}}{(k \cdot n)^{2}}\right) .
$$

The angular factor $2+k^{2} n^{2} /(k \cdot n)^{2}$ in Eq. (3.5) arises from the effective axial gauge propagator describing the quarkquark interaction, as in Eq. (2.31). Its presence makes the right-hand side of (3.5) ultraviolet convergent even if $Z$ and $m$ are constants. We will therefore look for approximate solutions of (3.5) with $Z=1$ and $m$ constant. Let us choose a coordinate system with $\mathbf{p}=0$, and let us take the string direction $n_{\mu}$ to be pure spacelike: $n_{\mu}=(0, \widehat{\mathbf{n}})$. Also, we want to carry out the calculation at finite temperature; therefore $\int d k$ means $(i / \beta) \sum_{n=-\infty}^{\infty} \int d^{3} \mathbf{k} /(2 \pi)^{3}$, and $k_{0}$ is to be replaced by $z_{n}=(2 \pi i / \beta) n$ in the integrand.

To evaluate (3.5) we first make use of the identity

$$
\hat{\mathbf{n}} \cdot \nabla_{k} \frac{1}{k^{2} \hat{\mathbf{n}} \cdot \mathbf{k}}=\frac{1}{k^{4}}\left(2-\frac{\hat{\mathbf{n}}^{2} k^{2}}{(\hat{\mathbf{n}} \cdot \mathbf{k})^{2}}\right),
$$

where $k^{2}=k_{0}^{2}-\mathbf{k}^{2}$. Using (3.6), and carrying out the partial integration, we obtain

$$
m=-\frac{e^{2} C_{F} m}{4 \pi^{2}}-2 e^{2} C_{F} m \frac{1}{\beta} \sum_{n=-\infty}^{\infty} \int \frac{d^{3} \mathbf{k}}{(2 \pi)^{3}} \frac{1}{z_{n}^{2}-\mathbf{k}^{2}} \frac{\partial}{\partial \mathbf{k}^{2}}\left(\frac{z_{n}^{2}-\mathbf{k}^{2}-\boldsymbol{M}^{2}-\boldsymbol{M}^{* 2}}{z_{n}^{2}-\mathbf{k}^{2}-\boldsymbol{M}^{* 2}} \frac{z_{n}^{2}-\mathbf{k}^{2}}{\left(p_{0}-z_{n}\right)^{2}-\mathbf{k}^{2}-m^{2}}\right) .
$$

The first term on the right-hand side of (3.7) is the surface term from the partial integration. Note that the dependence on $\hat{\mathbf{n}}$ has disappeared. Note also that the integral is well behaved both in the ultraviolet and in the infrared.

The perturbative vacuum exists only above the deconfinement temperature. In this regime we expect
$T \gg m, M^{*}$, because $M^{*}$ vanishes at $T=T_{c}$ (by definition of the deconfinement point) and the quark mass $m$ is much smaller than $T_{c}$. However, we also must have $T<M$, since only then can we expect the dual Lagrangian to be valid. Thus we want to evaluate (3.7) when $M \sim z_{n} \gg m, M^{*}$. Therefore, 


$$
\begin{aligned}
& m=-\frac{e^{2} C_{F} m}{4 \pi^{2}} \\
&-2 e^{2} C_{F} m \frac{1}{\beta} \sum_{n=-\infty}^{\infty} \int \frac{d^{3} \mathbf{k}}{(2 \pi)^{3}} \frac{1}{z_{n}^{2}-\mathbf{k}^{2}} \\
& \quad \times \frac{\partial}{\partial \mathbf{k}^{2}}\left(\frac{z_{n}^{2}-\mathbf{k}^{2}-M^{2}}{\left(p_{0}-z_{n}\right)^{2}-\mathbf{k}^{2}}\right) .
\end{aligned}
$$

It is shown in the Appendix that the right-hand side of (3.8) is negative. It is also (apart from the overall factor of $m$ ) independent of $m$. Therefore, for both of these reasons, the only solution is $m=0$ and consequently there is no chiral-symmetry breaking above the deconfinement temperature. This is a consequence of the properties of the perturbative vacuum propagators. In the nonperturbative vacuum, the presence of the magnetic condensate $\widetilde{F}_{0}^{2}$ modifies the low-momentum behavior of the propagators, and the Dyson equation has a chiralsymmetry-breaking solution with a scale determined by $\widetilde{F}_{0}^{2}$. We next show how this arises.

\section{CHIRAL-SYMMETRY BREAKING: THE NONPERTURBATIVE VACUUM}

The calculation described in Sec. III used the perturbative vacuum values for the dual gluon, $\widetilde{F}$, and mixed propagators. In the nonperturbative dual vacuum, characterized by a nonvanishing value of $\left\langle\widetilde{F}_{\mu \nu}^{a} \widetilde{F}_{\mu \nu}^{a}\right\rangle$, and in which confinement takes place, the propagators must be modified. To see how they are modified, we must look at the quadratic (in the $C$ and $\widetilde{F}$ fields) part of the dual QCD Lagrangian, where $\widetilde{F}^{2}$ is now measured from its nonzero nonperturbative value.

The Lagrangian is

$$
\begin{aligned}
\mathcal{L}= & \frac{M}{2} \widetilde{F}_{\mu \nu}^{a} G_{\mu \nu}^{a}+\frac{1}{4} \widetilde{F}_{\mu \nu}^{a} \mathcal{D}^{2}(C) \widetilde{F}_{\mu \nu}^{a} \\
& -\frac{1}{4} G_{\mu \nu}^{a} G_{\mu \nu}^{a}-W_{2}(\widetilde{F})-W_{4}(\widetilde{F}),
\end{aligned}
$$

where $G_{\mu \nu}^{a}=\partial_{\mu} C_{v}^{a}-\partial_{v} C_{\mu}^{a}+g f^{a b c} C_{\mu}^{b} C_{v}^{c}$, where $W_{2}$ and $W_{4}$ are the quadratic and quartic (in $\widetilde{F}$ ) parts of the "potential" $W(\widetilde{F})$. When $\widetilde{F}_{0}^{2}$ is introduced, the various inverse propagators will evidently all pick up terms proportional to $\widetilde{F}_{0}^{2}$. At very high momenta, these terms are unimportant; therefore, the asymptotic behavior of the propagators is the same as in the perturbative vacuum case, and the convergent ultraviolet behavior still holds. For momenta below $\left(-\widetilde{F}_{0}^{2}\right)^{1 / 2}$ (Ref. 7), however, the propagators behave like $1 / \widetilde{F}_{0}^{2}$ at large $\widetilde{F}_{0}^{2}$. Of the graphs shown in Fig. 5, therefore, all will behave like constants in the limit $\widetilde{F}_{0}^{2} / m^{2} \rightarrow \infty$, except the one associated with the four-quark vertex, labeled (b) in the figure. This graph contains no $C$ or $\widetilde{F}$ propagators. Its high loop momentum $\left(k^{2}>\widetilde{F}_{0}^{2}\right)$ contribution combines with that of the other graphs to produce the same ultraviolet convergence as in the perturbative vacuum, and all that survives in the limit $\widetilde{F}_{0}^{2} / \mathrm{m}^{2} \rightarrow \infty$ is its low loop momentum $\left(k^{2}<\widetilde{F}_{0}^{2}\right)$ contribution.

The net result is that in the nonperturbative vacuum, and for $\widetilde{F}_{0}^{2} / m^{2}$ large, the Dyson equation reduces to (again within the $m=$ const, $Z=1$ assumption)

$$
\begin{aligned}
m=-\frac{i e^{2} C_{F}}{2} \int & d k \frac{1}{4} \operatorname{Tr} \\
& \times\left(\frac{\epsilon_{\mu \nu \alpha \beta} n_{\alpha} \gamma_{\beta}}{k \cdot n} \frac{1}{\not p-\not k-m} \frac{\epsilon_{\mu v \gamma \delta} n_{\gamma} \gamma_{\delta}}{k \cdot n}\right),
\end{aligned}
$$

using the rules from Sec. II. The integral in (4.2) is to be cut off at $k^{2}=-\widetilde{F}_{0}^{2}$.

Working out the spinology yields an equation much like the gap equation in a superconductor: ${ }^{4}$ at $T=0$ we have

$m=-3 i e^{2} C_{F} m \int \frac{d^{4} k}{(2 \pi)^{4}} \frac{n^{2}}{(k \cdot n)^{2}} \frac{1}{(p-k)^{2}-m^{2}}$.

Evaluating the integral we find (setting $\mathbf{p}=0$ and choosing $n$ spacelike) either $m=0$ or

$$
1=\frac{3 e^{2} C_{F}}{8 \pi^{2}} \ln \left(-\widetilde{F}_{0}^{2} / m^{2}\right)
$$

from which we calculate the $T=0$ chiral-symmetrybreaking mass to be

$$
m^{2}=\left(-\widetilde{F}_{0}^{2}\right) e^{-8 \pi^{2} / 3 e^{2} C_{F}} .
$$

As in a superconductor, the mass is nonanalytic in the coupling constant. The role of the cutoff at the Debye energy is played by $\widetilde{F}_{0}^{2} \neq 0$; that is, when there is confinement, there is chiral-symmetry breaking.

Numerically, using the values of the parameters given in I, Eq. (4.5) gives a value of $100 \mathrm{MeV}$ for the zerotemperature mass, taking $\mathrm{SU}(3)$ as the color group. (This is, of course, a calculation with only a single quark flavor, so it is unclear what connection it has to any experimental quark masses.)

For finite temperatures, $\widetilde{F}_{0}^{2}$ and $m^{2}$ depend on $T$. If $\widetilde{F}_{0}^{2}(T) / m^{2}(T) \gg 1$, we can again approximate the Dyson equation by the gap equation, which at finite $T$ takes the form

$$
\begin{aligned}
& m(T)=3 e^{2} C_{F} m(T) \frac{1}{\beta} \\
& \times \sum_{n=-\infty}^{\infty} \int \frac{d^{3} \mathbf{k}}{(2 \pi)^{3}}\left[-\frac{1}{(\mathbf{k} \cdot \hat{\mathbf{n}})^{2}}\right] \\
& \quad \times \frac{1}{\left(p_{0}-z_{n}\right)^{2}-\mathbf{k}^{2}-m^{2}(T)},
\end{aligned}
$$

where $z_{n}-p_{0}=(2 \pi i / \beta)\left(n+\frac{1}{2}\right)$, and we have chosen $n_{v}$ to be pure spacelike. The temperature now provides an infrared cutoff in (4.6). The chiral transition temperature $T_{c}^{\prime}$, defined as the temperature for which the solution to (4.6) is $m\left(T_{c}^{\prime}\right)=0$, can be computed from

$$
1=3 e^{2} C_{F} \frac{1}{\beta_{c}^{\prime}} \sum_{n} \int \frac{d^{3} k}{(2 \pi)^{3}}\left(-\frac{1}{(\mathbf{k} \cdot \hat{\mathbf{n}})^{2}}\right) \frac{1}{\left(p_{0}-z_{n}\right)^{2}-\mathbf{k}^{2}} .
$$


Evaluating the sum and integral, with cutoff at $k=\left[-\widetilde{F}_{0}^{2}\left(T_{c}^{\prime}\right)\right]^{1 / 2}$, yields [for large $-\widetilde{F}_{0}^{2}\left(T_{c}^{\prime}\right)$ ]

$$
T_{c}^{\prime}=1.13\left[-\widetilde{F}_{0}^{2}\left(T_{c}^{\prime}\right)\right]^{1 / 2} e^{-4 \pi^{2} / 3 e^{2} C_{F}},
$$

in complete parallel to the usual superconductivity situation. ${ }^{4}$ If we replace $-\widetilde{F}_{0}^{2}$ by its value at $T=0$, and use values of the parameters from I, we obtain $T_{c}^{\prime} \sim 400 \mathrm{MeV}$.

In fact, however, $\widetilde{F}_{0}^{2}$ vanishes at the deconfinement temperature $T_{c}$. Both lattice estimates, ${ }^{8}$ and dual QCD estimates, ${ }^{9}$ of $T_{c}$ give a temperature well below $400 \mathrm{MeV}$; thus deconfinement occurs well below $T_{c}^{\prime}$. But once deconfinement occurs, and consequently $\widetilde{F}_{0}^{2}$ vanishes, we revert to the perturbative vacuum in which, as we have seen, there is no chiral-symmetry breaking. Thus chiral symmetry is restored at $T_{c}$, not at $T_{c}^{\prime}$.

\section{ACKNOWLEDGMENTS}

This work was supported in part by U.S. Department of Energy Contract No. DEAC06-81ER-40008 (M.B.), NSF Grant No. PHY-7812217 (J.S.B.), and U.S. Department of Energy Contract No. DEAC-03-81ER40050 (F.Z.).

\section{APPENDIX}

In Sec. II we showed that, in the perturbative vacuum, the effective interaction between quarks is given by Eq. (2.31). In the nonperturbative vacuum, the coefficient in
(2.31) will change, and the other possible spinological invariant, $\delta_{\mu v}-n_{\mu} n_{v} / n^{2}$, will appear as well. The form of the Dyson equation in the nonperturbative vacuum will therefore be (as before, setting $Z=1$ and taking $m$ to be constant)

$$
\begin{gathered}
m=-i e^{2} C_{F} m \int d k\left[F\left(k^{2}\right)+G\left(k^{2}\right) \frac{n^{2}}{(n \cdot k)^{2}}\right] \\
\times \frac{1}{(p-k)^{2}-m^{2}} .
\end{gathered}
$$

In the perturbative vacuum, $G=\left(k^{2} / 2\right) F$, so that only the structure $2+n^{2} k^{2} /(n \cdot k)^{2}$ appears, as indicated in Eq. (3.5); in the nonperturbative case we write more generally

$$
G=\frac{k^{2}}{2} F+3 H
$$

[The factor of 3 results from $\gamma_{\mu}\left(\delta_{\mu \nu}-n_{\mu} n_{\nu} / n^{2}\right) \gamma_{\nu}=3$.] As we will see below, $H$ is essential for chiral-symmetry breaking to take place.

Let us first look at zero temperature, in which case we may set $p=0$ in (A1), and $d k$ stands for $d^{4} k /(2 \pi)^{4}$. We note that, choosing $n_{\mu}$ to be pure spacelike,

$$
\frac{1}{(\hat{\mathbf{n}} \cdot \mathbf{k})^{2}}=-\hat{\mathbf{n}} \cdot \nabla_{k} \frac{1}{\hat{\mathbf{n}} \cdot \mathbf{k}} \text {. }
$$

Using (A3) in (A1) and performing the indicated partial integration yields

$$
m=-i e^{2} C_{F} m\left[\frac{1}{2 \pi^{2}} \int_{-\infty}^{\infty} \frac{d k_{0}}{2 \pi} \lim _{|\mathbf{k}| \rightarrow \infty} \frac{G\left(k_{0}^{2}-\mathbf{k}^{2}\right)}{k_{0}^{2}-\mathbf{k}^{2}-m^{2}}+\int_{-\infty}^{\infty} \frac{d k_{0}}{2 \pi} \int \frac{d^{3} \mathbf{k}}{(2 \pi)^{3}}\left[\frac{F\left(k^{2}\right)}{k^{2}-m^{2}}-2 \frac{\partial}{\partial \mathbf{k}^{2}} \frac{G\left(k^{2}\right)}{k^{2}-m^{2}}\right]\right]
$$

For very large $k^{2}$, the nonperturbative vacuum propagators coincide with those in the perturbative vacuum. Therefore, as $k^{2} \rightarrow \infty, F \rightarrow 2 / k^{2}, G \rightarrow 1$, and $H \rightarrow 0$. All are finite at the origin in the nonperturbative vacuum. The surface term in (A4) can therefore be evaluated explicitly and contributes $-e^{2} C_{F} m / 4 \pi^{2}$ to the right-hand side.

We next carry out the Wick rotation to Euclidean space, $k_{0} \rightarrow i k_{4}$, and invoke (A2) to obtain

$$
\begin{aligned}
m=-\frac{e^{2} C_{F} m}{4 \pi^{2}}+\frac{e^{2} C_{F}}{16 \pi^{2}} \int_{0}^{\infty} k_{E}^{2} d k_{E}^{2} & \\
\times & \left(\frac{F}{k_{e}^{2}+m^{2}}+\frac{d}{d k_{E}^{2}} \frac{k_{E}^{2} F}{k_{E}^{2}+m^{2}}\right. \\
& \left.-6 \frac{d}{d k_{E}^{2}} \frac{H}{k_{E}^{2}+m^{2}}\right),
\end{aligned}
$$

where $k_{E}^{2}=k_{4}^{2}+\mathbf{k}^{2}$. Because

$$
\frac{k_{E}^{2} F}{k_{E}^{2}+m^{2}}+k_{E}^{2} \frac{d}{d k_{E}^{2}} \frac{k_{e}^{2} F}{k_{E}^{2}+m^{2}}=\frac{d}{d k_{E}^{2}} \frac{k_{E}^{2} F}{k_{E}^{2}+m^{2}},
$$

we can finally rewrite (A5) as

$$
m=-\frac{e^{2} C_{F} m}{4 \pi^{2}}+\frac{e^{2} C_{F} m}{8 \pi^{2}}+\frac{3 e^{2} C_{F} m}{8 \pi^{2}} \int_{0}^{\infty} d k_{E}^{2} \frac{H\left(-k_{E}^{2}\right)}{k_{E}^{2}+m^{2}},
$$

using the fact that $F \rightarrow 2 / k^{2}$ and $H \rightarrow 0$ as $k^{2} \rightarrow \infty$. The first two terms in (A7) come from the known large- $k^{2}$ behavior of $F$ and $H$. The third term, giving the contribution of finite momenta to $m$, depends only on $H$.

In the perturbative vacuum, $H=0$. Hence, the only solution of (A7) is $m=0$ and chiral symmetry remains unbroken.

In the nonperturbative vacuum, where $\widetilde{F}_{0}^{2} \neq 0$, the contributions of $H$ to (A7) arise from momenta below or of order $\left(-\widetilde{F}_{0}^{2}\right)^{1 / 2}$. As explained in the text we can use the graphs of Fig. 5 to estimate the contribution when $-\widetilde{F}_{0}^{2} / m^{2} \gg 1$. This yields

$$
m=-\frac{e^{2} C_{F} m}{8 \pi^{2}}+\frac{3 e^{2} C_{F} m}{8 \pi^{2}} \int_{0}^{-\tilde{F}_{0}^{2}} \frac{d k_{E}^{2}}{k_{E}^{2}+m^{2}},
$$

which reproduces Eq. (4.4) in the limit of large $\widetilde{F}_{0}^{2}$.

We now look at finite temperature, and replace $\int d k$ in 
(A1) by $(i / \beta) \Sigma_{n} \int d^{3} k /(2 \pi)^{3}$. We also set $\mathbf{p}=0$, $p_{0}=(2 \pi i / \beta)\left(n_{0}+\frac{1}{2}\right)$, and $k_{0}=(2 \pi i / \beta) n$. To explicitly carry out the integrals let us write $F$ as a sum of terms,

$$
F=\sum_{i} \frac{2 \lambda i}{k^{2}-m_{i}^{2}}
$$

with $\sum_{i} \lambda_{i}=1$ to incorporate the known asymptotic behavior. [Any rational function of $k^{2}$, such as, for example, the one given in (2.31), can be written this way.] It is then a straightforward calculation to show that (A1) reduces to

$m=-\frac{e^{2} C_{F} m}{4 \pi^{2}} \int_{0}^{\infty} d k\left[\frac{\tanh \beta E / 2}{E}+\sum_{i} \lambda_{i}\left(2 k^{2}+m_{i}^{2}\right)\left[\frac{A}{A^{2}-4 p_{0}^{2} E_{i}^{2}} \frac{\tanh \beta E / 2}{E}+\frac{B}{B^{2}-4 p_{0}^{2} E^{2}} \frac{\operatorname{coth} \beta E_{i} / 2}{E_{i}}\right]\right]$

where $E^{2}=k^{2}+m^{2}, \quad E_{i}^{2}=k^{2}+m_{i}^{2}, \quad A=p_{0}^{2}+m^{2}-m_{i}^{2}$, and $B=p_{0}^{2}-m^{2}+m_{i}^{2}$, plus the contribution of the $H$ term.

At high $T$ or small $\beta$, and therefore high $p_{0}$ (which is the relevant range), the first term in (A10) dominates and it has a negative sign. Hence in the perturbative vacuum, where $H=0$, the only solution to (A10), at least for large
$T$, is $m=0$ and therefore there is no chiral-symmetry breaking.

In the nonperturbative vacuum, we can again use the graphs of Fig. 5 to estimate the contribution of $\boldsymbol{H}$. Adding this to (A10) then yields Eq. (4.8) in the limit of large $\widetilde{F}_{0}^{2}$.
${ }^{1}$ M. Baker, J. Ball, and F. Zachariasen, Phys. Rev. D 37, 1036 (1988); 37, 3785(E) (1988); hereafter referred to as $I$.

${ }^{2}$ J. Ball and F. Zachariasen, Phys. Lett. 106B, 133 (1981); J. M. Cornwall, Phys. Rev. D 22, 1452 (1980).

${ }^{3}$ R. Gupta et al., Phys. Rev. Lett. 57, 2621 (1986); F. Karsch, J. B. Kogut, D. K. Sinclair, and H. W. Wyld, Phys. Lett. B 188, 353 (1987).

${ }^{4}$ See, for example, A. Fetter and J. D. Walecka, Quantum Theory of Many Particle Systems (McGraw-Hill, New York, 1971), p. 447.

${ }^{5}$ F. Englert, in Hadron Structure and Lepton-Hadron Interac- tions, Cargèse, 1977, edited by M. Lévy et al. (Plenum, New York, 1979), pp. 503-560.

${ }^{6}$ Note that with $n_{\mu}$ present, the form (3.2) for $S$ is not strictly valid. There can be further invariants. All dependence on $n$, of course, disappears in $\langle\bar{q} q\rangle$, which is a gauge-invariant quantity. We will ignore $n$-dependent invariants in $S$.

${ }^{7}$ Recall that $\widetilde{F}_{0}^{2}<0$, as appropriate for a magnetic condensate.

${ }^{8}$ R. Gupta et al., Phys. Lett. B 201, 523 (1988); J. B. Kogut et al., Phys. Rev. Lett. 55, 1475 (1985).

${ }^{9}$ M. Baker, J. Ball, and F. Zachariasen, Phys. Rev. Lett. 61, 521 (1988). 